\title{
Prevalence, Risk Factors, and Psychological Effects of Primary Nocturnal Enuresis in Chinese Young Adults
}

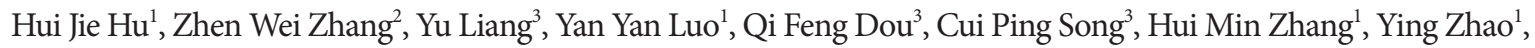 \\ Guang Run Tian ${ }^{3}$, Ke Zhang ${ }^{3}$, Qiu Fang Mao², Jing Gui Song', Soren Rittig ${ }^{5}$, Jian Guo Wen ${ }^{3,4,6}$ \\ ${ }^{1}$ Nursing School, ${ }^{2}$ Third, ${ }^{3}$ First, and ${ }^{4}$ Second Affiliated Hospital of Xinxiang Medical University, Xinxiang, China \\ ${ }^{5}$ Department of Paediatrics, Aarhus University Hospital, Aarhus, Denmark \\ ${ }^{6}$ Henan Joint International Pediatric Urodynamic Laboratory, Zhengzhou University, Zhengzhou, China
}

Purpose: This study aimed to investigate the prevalence, risk factors, and effects of primary nocturnal enuresis (PNE) on physical and mental health in young adults in mainland China.

Methods: An anonymous questionnaire was used to collect information including the sociodemographic characteristics, history of PNE, family history, daytime voiding symptoms, Pittsburgh Sleep Quality Index (PSQI) scores, Self-Esteem Scale (SES), and Self-Rating Depression Scale (SDS). A total of 22,500 university students from 23 provinces and 368 cities in mainland China were included.

Results: In total, 21,082 questionnaires were collected, and 20,345 of them qualified for statistical analysis. The PNE prevalence was $1.17 \%$, and the distribution of monosymptomatic nocturnal enuresis (MNE) and nonmonosymptomatic nocturnal enuresis (NMNE) was $66.1 \%$ and 33.9\%, respectively. In total, $28 \%$ of respondents with PNE reported bedwetting daily, $31.6 \%$ between 1 and 7 times weekly, and 40.4\% between 1 and 4 times monthly; 80\% of PNE cases had no history of treatment. The prevalence of PNE in patients with a family history, frequency, urgency, urinary incontinence, and recurrent urinary tract infections was significantly higher than in those without these conditions $(\mathrm{P}<0.001)$. PNE was significantly correlated with the PSQI total score (sleep quality) $(\mathrm{P}=0.011)$. The SES score was lower and the SDS was higher $(\mathrm{P}<0.001)$ in the PNE group than in those without PNE.

Conclusions: In mainland China, the PNE prevalence among young adults was found to be high, and PNE had significant effects on physical and mental health. Risk factors included a family history, daytime voiding symptoms, and lack of treatment.

Keywords: Primary nocturnal enuresis; Prevalence; Risk factors; Young adults; Mental health

- Fund/Grant Support: This study was supported by Henan Province Medical Science Provincial-ministerial Construction Project (No: SBGJ2018059); National Nature Science Foundation of China (No. U1904208,81670689); and Henan International Cooperative Project (No:182102410002).

- Research Ethics: This study was approved by the Xinxiang Medical University Ethics Committee (XYLL-2020232). The subjects were fully informed of the content and information of the survey. Subjects provided written informed consent during the process of survey completion. To protect the rights and interests of the subjects, this research was conducted in accordance with the Helsinki Declaration as revised in 1989.

- Conflict of Interest: No potential conflict of interest relevant to this article was reported.

Corresponding author: Jian Guo Wen (iD https://orcid.org/0000-0003-0952-118X The First Affiliated Hospital of Xinxiang Medical University, No. 88, Weihui, Xinxiang 453003 Henan, China

Email: wenjg@hotmail.com

Submitted: August 18, 2020 / Accepted after revision: September 29, 2020
This is an Open Access article distributed under the terms of the Creative Commons Attribution Non-Commercial License (https://creativecommons.org/licenses/by-nc/4.0/) which permits unrestricted non-commercial use, distribution, and reproduction in any medium, provided the original work is properly cited. 


\section{INTRODUCTION}

Nocturnal enuresis (NE) is both a symptom and a condition of intermittent incontinence that occurs during periods of sleep; the symptom of incontinence requires a minimum age of 5 years, a minimum of 1 episode per month, and a minimum duration of 3 months to be termed a condition. Primary nocturnal enuresis (PNE) is defined as above in a child that was never "dry" for longer than 6 consecutive months [1-3]. It is well known that the prevalence of PNE decreases with age, but it does not disappear in adolescents and adults. However, the reported prevalence of PNE among adolescent and adult populations is inconsistent, with large variations across studies, and has been reported to range from $0.3 \%$ to $6.0 \%$ [4-10].

It has been reported that many factors, such as sex, family history, place of residence (living in a rural or urban area), and arousal difficulty, are involved in the development of PNE [1113]. From an etiological perspective, a large percentage of adolescent and adult PNE cases are thought to represent patients in whom pediatric PNE persists. Other factors involved in the pathophysiology of adolescent and adult PNE have also been considered, such as various medical problems or underlying urological diseases.

In our previous studies, the PNE prevalence decreased with age, decreasing from $11.83 \%$ and $15.13 \%$ in 5-year-old subjects to $1.05 \%$ and $1.13 \%$ in 18 -year-old subjects in 2006 and 2017 , respectively $[8,9]$. However, the PNE prevalence in mainland China and its risk factors after 18 years of age are unclear. In addition, PNE is known to cause serious psychological problems and substantial burdens and life difficulties for children and their families. Undoubtedly, if PNE continues into adulthood, physical and mental health may be seriously influenced [14,15]. However, the mental effects of PNE on Chinese young adults have rarely been reported. Therefore, it is necessary to explore the prevalence, risk factors, and mental effects of PNE in these young adults.

Considering their level of cooperation and ability to understand the questionnaires, university students are reasonable candidates to survey. We hypothesized that the prevalence of PNE and its effects on mental health in young adults would be high and that their risk factors might be different from those in children. Consequently, the purpose of this study is to determine the prevalence of PNE, its risk factors, and its effects on mental health in young adults through a large-sample assessment in mainland China. This study provides novel informa- tion for a further understanding of the characteristics of PNE in young adults and its impact on mental health.

\section{MATERIALS AND METHODS}

\section{Study Participants}

From March 2019 to October 2019, we investigated 22,500 university students who came from 23 provinces and 368 cities all over China. This study was approved by the Xinxiang Medical University Ethics Committee (XYLL-2020232). The subjects were fully informed of the content and information of the survey. Subjects provided written informed consent during the process of survey completion. To protect the rights and interests of the subjects, this research was conducted in accordance with the Helsinki Declaration as revised in 1989.

\section{Content of the Questionnaire}

An anonymous questionnaire was used to collect information. A survey was conducted on the Chinese mainland using internet and on-the-spot questionnaires, which were distributed by professionally trained investigators who explained the purpose and significance of the survey to students. After respondents filled out the questionnaire, it was placed in a sealed questionnaire box to maximize students' privacy. A self-administered questionnaire comprising 2 sections required boxes to be ticked. The first section was used to document the background information of the student, such as date of birth, sex, and place of residence (living in a rural or urban area). The second part included questions about the details of bedwetting and relevant factors, including: (1) whether bedwetting had continued from birth to now; (2) PNE frequency; (3) PNE family history (i.e., any member of the family having a history of enuresis; this parameter was measured as a proxy for genetic causes of PNE, and if the respondent did not know whether he or she had a family history of enuresis, the respondent was asked to consult his or her relatives); (4) ease of waking at night; (5) previous treatment; (6) whether the respondent experienced frequency, urgency, or urinary incontinence (UI) during the daytime; and 7) whether a urinary tract infection (UTI) was present, now or in the past.

The questionnaire also contained 3 other instruments. First, the Pittsburgh Sleep Quality Index (PSQI) score was used to evaluate sleep quality and disturbance over the past month. The PSQI contains 19 items. In brief, the first 4 items are open questions ("What time do you usually go to bed?", "How long does 
it take you to fall asleep?", "What time do you usually get up in the morning?", and "How long do you actually sleep at night?"). Then, items 5-19 are rated on a 4-point scale. The item scores yielded the following 7 subscores ranging from 0 to 3 : $\mathrm{C} 1$ (sleep quality), C2 (sleep latency), C3 (sleep duration), C4 (sleep efficiency), C5 (sleep disturbance), C6 (sleep medication use), and C7 (daytime dysfunction due to sleepiness). The total score, which ranges from 0 to 21 , is obtained by summing the 7 subscores. A higher score corresponds to poorer subjective sleep quality. Chinese researchers found that the Cronbach alpha coefficient of the PSQI was 0.734 [16]. Next, the Rosenberg SelfEsteem Scale (SES) was used to measure self-esteem. The scale consists of 10 items rated on a 4-point Likert scale with options ranging from 1 (strongly disagree) to 4 (strongly agree). The total score ranges from 10 to 40 , with a higher score indicating higher self-esteem [17]. The SES has shown good reliability and validity for measuring self-esteem [18]. In our study, the Cronbach alpha coefficient of the SES was 0.797. Finally, the survey also contained the Self-Rating Depression Scale (SDS), which was developed by Zung (Cronbach alpha coefficient $=0.920$; $\mathrm{P}<0.001$ ) [19]. This scale is one of the most commonly used and convenient psychological measurement tools. The SDS is a self-rated scale consisting of 20 questions, including questions addressing psycho-emotional, physical, and psychomotor disorders. The score for each question ranges from 1 to 4 , and the total score for each item is the original score, which is multiplied by 1.25 to obtain the standard score. The standard score for depression is 50 and a higher score corresponds to a greater likelihood of depression.

\section{Definitions and Exclusion Criteria}

$\mathrm{NE}$ is both a symptom and a condition of intermittent incontinence that occurs during periods of sleep; the symptom of in- continence requires a minimum age of 5 years, a minimum of 1 episode per month and a minimum duration of 3 months to be termed a condition. Primary NE (never dry) and secondary NE (defined arbitrarily as a relapse after a dry period of 6 months or longer) as well as monosymptomatic NE (MNE, no lower urinary tract symptoms) and nonmonosymptomatic NE (NMNE, with lower urinary tract symptoms) have been widely used to describe NE [1-3]. The PNE criterion in this study was in accordance with the definition of the International Children Continence Society (never "dry" for longer than 6 consecutive months) [3]. The exclusion criteria were obvious organic diseases, such as lower bladder urinary tract obstruction, neurological diseases, and wetting recurrence after a dry period longer than 6 months.

\section{Statistical Analysis}

The data set was established using the Epidata software (ver. 3.1, Odense, Denmark). Statistical analyses were performed using IBM SPSS Statistics ver. 21.0 (IBM Co., Armonk, NY, USA). Variables including sex, residence, frequency, UI, urgency, UTI, and family history of PNE were compared using the chi-square test. Logistic regression was used for multivariate analysis. The Mann-Whitney U-test was used to compare SDS and SES scores between the PNE and non-PNE groups. A P-value $<0.05$ was considered to indicate statistical significance. Associations between PNE and sleep quality (PSQI scores C1-7 and the total score) were analyzed by Pearson correlation tests.

\section{RESULTS}

\section{Prevalence of PNE}

A total of 21,082 questionnaires were collected, of which 20,345 (89.3\%) (respondents' age, 17-21 years; 6,583 men and 13,762

Table 1. Comparison of prevalence rates in different age groups

\begin{tabular}{|c|c|c|c|c|c|c|c|c|}
\hline \multirow{2}{*}{ Age (yr) } & \multicolumn{2}{|c|}{ Male } & \multicolumn{2}{|c|}{ Female } & \multicolumn{2}{|c|}{ Total } & \multirow{2}{*}{$x^{2}$} & \multirow{2}{*}{ P-value } \\
\hline & No. & PNE & No. & PNE & No. & PNE & & \\
\hline 17 & 1,293 & $14(1.08)$ & 2,591 & $27(1.04)$ & 3,884 & $41(1.06)$ & 0.01 & 0.91 \\
\hline 18 & 2,017 & $32(1.59)$ & 3,772 & $42(1.11)$ & 5,789 & $74(1.28)$ & 2.33 & 0.13 \\
\hline 19 & 1,765 & $22(1.25)$ & 3,285 & $34(1.04)$ & 5,050 & $56(1.11)$ & 0.47 & 0.49 \\
\hline 20 & 733 & $10(1.36)$ & 1,893 & $24(1.27)$ & 2,626 & $34(1.29)$ & 0.04 & 0.85 \\
\hline 21 & 775 & $10(1.29)$ & 2,221 & $24(1.08)$ & 2,996 & $34(1.13)$ & 0.25 & 0.62 \\
\hline Total & 6,583 & $88(1.34)$ & 13,762 & $151(1.10)$ & 20,345 & $239(1.17)$ & 2.20 & 0.13 \\
\hline
\end{tabular}

Values are presented as number (\%).

PNE, primary nocturnal enuresis. 
women) of which qualified for statistical analysis. The overall prevalence of PNE was $1.17 \%$, and the prevalence was $1.3 \%$ in men and $1.1 \%$ in women. No significant difference was found between men and women in any age groups $(\mathrm{P}>0.05)$ (Table 1$)$. The prevalence of PNE among different age groups ranged from $1.04 \%$ to $1.59 \%$. MNE and NMNE accounted for $66.1 \%$ (158 of 239) and 33.9\% (81 of 239) of all PNE cases, respectively. A total of $28 \%$ respondents with PNE (67 of 239) reported bedwetting daily, 31.6\% (76 of 239) reported bedwetting between 1 and 7 times per week, and 40.4\% (96 of 239) reported bedwetting between 1 and 4 times monthly. Most of the respondents with PNE (80\%) had no previous history of treatment.

\section{Risk Factors for PNE}

Univariate analysis showed no significant difference in the prevalence of PNE between men and women or between urban and rural areas $(\mathrm{P}>0.05)$. The prevalence of PNE in young adults with a family history; a history of UTI; current UTI; and urinary frequency, urgency, and UI was higher than that among normal young adults $(\mathrm{P}<0.001)$ (Table 2$)$.

The results of the multivariate logistic regression analysis are shown in Table 3. A family history (odds ratio [OR], 5.043; 95\% confidence interval [CI], 2.962-8.586), past history of UTI (OR, 2.462; 95\% CI, 1.275-4.754), current UTI (OR, 7.261; 95\% CI, 3.996-13.196), urinary frequency (OR, 1.889; 95\% CI, $1.344-$ 2.655), urgency (OR, 1.658; 95\% CI, 1.194-2.304), and UI (OR,

Table 2. The prevalence and risk factors for PNE in young adults

\begin{tabular}{|c|c|c|c|c|c|}
\hline \multirow{2}{*}{ Risk factor } & \multirow{2}{*}{ No. } & \multicolumn{2}{|c|}{ PNE } & \multirow{2}{*}{$x^{2}$} & \multirow{2}{*}{ P-value } \\
\hline & & Yes & No & & \\
\hline \multicolumn{6}{|l|}{ Sex } \\
\hline Male & 6,583 & $88(1.3)$ & $6,495(98.7)$ & 2.20 & 0.13 \\
\hline Female & 13,762 & $151(1.1)$ & $9,022(98.9)$ & & \\
\hline \multicolumn{6}{|c|}{ Place of residence } \\
\hline Urban & 7,247 & $79(1.0)$ & $7,168(99.0)$ & 0.694 & 0.405 \\
\hline Rural & 13,098 & $160(1.2)$ & $12,938(98.8)$ & & \\
\hline \multicolumn{6}{|c|}{ Family history } \\
\hline Yes & 637 & $81(12.7)$ & $565(87.3)$ & 742.54 & $<0.001$ \\
\hline No & 19,708 & $158(0.8)$ & $19,550(99.2)$ & & \\
\hline \multicolumn{6}{|c|}{ Past history of UTI } \\
\hline Yes & 1,365 & $71(5.2)$ & $1,294(94.8)$ & 202.71 & $<0.001$ \\
\hline No & 18,870 & $168(0.9)$ & $18,702(99.1)$ & & \\
\hline \multicolumn{6}{|c|}{ Current UTI } \\
\hline Yes & 921 & $70(7.6)$ & $851(82.4)$ & 343.09 & $<0.001$ \\
\hline No & 19,424 & $169(0.9)$ & $19,255(99.1)$ & & \\
\hline \multicolumn{6}{|l|}{ Frequency } \\
\hline Yes & 3,171 & $111(3.5)$ & $3,060(96.4)$ & 175.02 & $<0.001$ \\
\hline No & 17,174 & $128(0.8)$ & $17,046(99.2)$ & & \\
\hline \multicolumn{6}{|l|}{ Urgency } \\
\hline Yes & 4,833 & $116(2.4)$ & 4,717 (97.6) & 81.993 & $<0.001$ \\
\hline No & 15,512 & $123(0.8)$ & $15,389(99.2)$ & & \\
\hline \multicolumn{6}{|l|}{ UI } \\
\hline Yes & 2,290 & $126(5.5)$ & $2,164(94.5)$ & 413.08 & $<0.001$ \\
\hline No & 17,945 & $113(0.6)$ & $17,832(99.4)$ & & \\
\hline
\end{tabular}

Values are presented as number (\%).

PNE, primary nocturnal enuresis; UTI, urinary tract infection; UI, urinary incontinence. 
Table 3. Logistic regression analysis of risk factors of PNE prevalence

\begin{tabular}{|c|c|c|c|c|c|}
\hline Risk factor & $\beta$ & SE & P-value & OR & $95 \% \mathrm{CI}$ \\
\hline Family history & 1.618 & 0.271 & $<0.001$ & 5.043 & $2.962-8.586$ \\
\hline Past history of UTI & 0.901 & 0.336 & 0.007 & 2.462 & $1.275-4.754$ \\
\hline Current UTI & 1.983 & 0.305 & $<0.001$ & 7.261 & $3.996-13.196$ \\
\hline Frequency & 0.636 & 0.174 & $<0.001$ & 1.889 & $1.344-2.655$ \\
\hline Urgency & 0.506 & 0.168 & 0.003 & 1.658 & $1.194-2.304$ \\
\hline UI & 1.824 & 0.168 & $<0.001$ & 6.198 & $4.460-8.613$ \\
\hline
\end{tabular}

PNE, primary nocturnal enuresis; UTI, urinary tract infection; UI, urinary incontinence; OR, odds ratio; CI, confidence interval.

Table 4. SES and SDS scores between the PNE and non-PNE groups

\begin{tabular}{|c|c|c|c|c|c|}
\hline \multirow{2}{*}{ Score } & \multirow{2}{*}{ Sex } & \multicolumn{2}{|c|}{ PNE } & \multirow{2}{*}{$z$} & \multirow{2}{*}{ P-value } \\
\hline & & Yes & No & & \\
\hline \multirow[t]{3}{*}{ SES } & Male & $27.03 \pm 6.04$ & $29.34 \pm 5.59$ & 2.79 & 0.005 \\
\hline & Female & $27.21 \pm 3.02$ & $29.54 \pm 4.10$ & 2.12 & 0.034 \\
\hline & Total & $27.02 \pm 5.36$ & $29.37 \pm 4.96$ & 3.48 & $<0.001$ \\
\hline \multirow[t]{3}{*}{ SDS } & Male & $53.09 \pm 13.30$ & $45.42 \pm 12.57$ & -3.79 & $<0.001$ \\
\hline & Female & $56.43 \pm 6.81$ & $47.61 \pm 9.34$ & -3.52 & $<0.001$ \\
\hline & Total & $53.25 \pm 11.74$ & $45.62 \pm 11.27$ & -4.98 & $<0.001$ \\
\hline
\end{tabular}

Values are presented as mean \pm standard deviation.

PNE, primary nocturnal enuresis; SES, Self-Esteem Scale; SDS, Self-Rating Depression Scale.

Higher scores indicate higher self-esteem and greater depression.

Table 5. The effects of PNE on sleep quality

\begin{tabular}{|c|c|c|c|c|c|c|}
\hline \multirow{2}{*}{ PSQI score } & \multicolumn{2}{|c|}{ Male } & \multicolumn{2}{|c|}{ Female } & \multicolumn{2}{|c|}{ Total } \\
\hline & $\mathrm{r}$ & $\mathrm{P}$-value & $\mathrm{r}$ & P-value & $\mathrm{r}$ & P-value \\
\hline C1 (sleep quality) & $0.080^{\star *}$ & 0.005 & 0.01 & 0.005 & 0.026 & 0.001 \\
\hline C2 (sleep latency) & 0.009 & 0.742 & 0.020 & 0.341 & 0.017 & 0.288 \\
\hline C3 (sleep duration) & 0.043 & 0.130 & 0.011 & 0.594 & 0.031 & 0.057 \\
\hline C4 (sleep efficiency) & 0.000 & 0.993 & 0.00 & 0.815 & 0.003 & 0.871 \\
\hline C5 (sleep disturbance) & $0.271^{\star *}$ & 0.019 & $0.330^{*}$ & 0.013 & $0.341^{\star}$ & 0.000 \\
\hline C6 (sleep medication use) & $0.126^{\star *}$ & 0.000 & $0.089^{* *}$ & 0.000 & $0.119^{\star *}$ & 0.000 \\
\hline C7 (daytime dysfunction) & $0.093^{* *}$ & 0.001 & 0.086 & 0.003 & $0.035^{\star}$ & 0.034 \\
\hline Total score & $0.098^{\star \star}$ & 0.001 & 0.067 & 0.009 & $0.041^{\star}$ & 0.011 \\
\hline
\end{tabular}

PNE, primary nocturnal enuresis; PSQI, Pittsburgh Sleep Quality Index.

' $r$ ' corresponds to Pearson correlation coefficients.

${ }^{\star}$ Correlation is significant at the 0.05 level (2-tailed). ${ }^{*}$ Correlation is significant at the 0.01 level (2-tailed).

6.198; 95\% CI, 4.460-8.613) significantly increased the prevalence of PNE.

\section{Effects of PNE on SES, SDS, and PSQI Scores}

The SES scores were significantly lower in subjects with PNE than in those without $\operatorname{PNE}(\mathrm{z}=3.48, \mathrm{P}<0.001)$. The depression scale score was higher in the PNE group than in the non-PNE group $(z=-4.98, P<0.001)$ (Table 4$)$. The effects of PNE on sleep quality, which were analyzed using Pearson correlation coefficients, are shown in Table 5. In all university students, the 
Table 6. Differences in PSQI, SES, and SDS between the MNE and NMNE groups

\begin{tabular}{lcccc}
\hline Total score & MNE $(\mathrm{n}=157)$ & NMNE $(\mathrm{n}=82)$ & $z$ & P-value \\
\hline SES & $29.60 \pm 4.21$ & $27.42 \pm 3.23$ & 1.699 & 0.008 \\
SDS & $47.24 \pm 9.87$ & $52.77 \pm 8.02$ & 1.515 & 0.038 \\
PSQI & $4.23 \pm 2.03$ & $4.45 \pm 2.57$ & 1.603 & 0.012 \\
\hline
\end{tabular}

Values are presented as mean \pm standard deviation.

PSQI, Pittsburgh Sleep Quality Index; SES, Self-Esteem Scale; SDS, Self-Rating Depression Scale; MNE, monosymptomatic nocturnal enuresis; NMNE, nonmonosymptomatic nocturnal enuresis.

Higher scores indicate higher self-esteem, greater depression, and poor sleep quality.

total PSQI, sleep quality, sleep medication use, and daytime dysfunction scores were significantly correlated with PNE $(\mathrm{P}<0.05)$. However, no significant correlations were found between sleep latency, sleep duration, and sleep efficiency and PNE $(\mathrm{P}>0.05)$. A significant correlation was identified between sleep disturbance and PNE in men $(r=0.271, P=0.019)$. Additionally, a significant correlation was found between sleep disturbance and PNE in women $(\mathrm{r}=0.330, \mathrm{P}=0.013)$. The SES scores of the NMNE group were significantly lower than that of the MNE group $(\mathrm{z}=1.699, \mathrm{P}=0.008)$. The SDS scores were higher in the NMNE group than in the MNE group $(z=1.515$, $\mathrm{P}=0.038$ ). For the PSQI, the total scores were significantly higher in the NMNE group than in the MNE group $(z=1.603$, $\mathrm{P}=0.012)$ (Table 6).

\section{DISCUSSION}

PNE is common in clinical practice and causes a significant social burden in both Eastern and Western countries. Many people who experience PNE continue bedwetting when they grow up with no clear reason although it has been known that many factors contribute to this abnormality. With social and economic development, there is an increasing need to further understand and prevent PNE in adults [20].

The overall prevalence of PNE, as well as its prevalence in different age groups, varies substantially in different countries. In the published literature, the reported prevalence of PNE in adolescents and adults varies significantly [4-10]. This might be due to differences in survey selection area, geographical environment, PNE definition, the survey implementation method (selfcompleted questionnaire, mailed questionnaire, telephone follow-up, or personal face-to-face surveys), and characteristics of the survey population. In addition, the prevalence of MNE and NMNE is rarely reported. Survey documentation of PNE in young adults in mainland China is still lacking. Therefore, the present study surveyed the prevalence and risk factors of PNE in the population of university students in mainland China (as outstanding representatives of young adults), with special attention paid to its effects on mental health.

More than $48.1 \%$ of young adults in mainland China attend universities. University students clearly account for an important part of Chinese youth, and are also well-educated, enabling them to better understand the questionnaires, thereby increasing the credibility of the survey. More importantly, university students have higher self-esteem and other self-image attributes. According to our epidemiological studies among children and adolescents, an estimated $1.0 \%$ to $1.5 \%$ of university students in mainland China have PNE [8]. A survey of 15,000 university students is sufficient for data analysis. To further reduce the error and increase the authenticity and reliability of the data, we investigated 22,500 university students. In addition, the university students in our survey were from different provinces across the country, which helped to determine the prevalence in this age group more accurately. In the present crosssectional study, the overall prevalence of PNE in young adults was $1.17 \%$, which is similar to the prevalence reported in the same age group in the literature. Moreover, the present study analyzed both the overall prevalence and that of MNE and NMNE, as well as the distribution of PNE severity in young adults.

The prevalence rates of PNE among men and women in this survey were $1.3 \%$ and $1.1 \%$, respectively, with no significant difference between the sexes. This is similar to research performed in 21- to 25-year-olds in Hong Kong [4]. The prevalence of PNE did not decrease with age, which indicates that bedwetting in young adults becomes stable and implies that more active interventions should be considered, rather than continuing to await the development of voiding control. No significant difference in the prevalence of PNE was found between residents of urban and rural areas, which might be explained by 
the fact that the gap between living conditions in urban and rural areas has significantly decreased with economic development in the last 20 years in mainland China.

Most cases of enuresis in children are MNE [21]. Similarly, the present study found that MNE accounted for $66.1 \%$ of cases in young adults. A survey in Egypt showed that UTIs and pinworm infestations occurred at high rates in children with MNE [22]. A survey in Pakistan reported that the incidence of NE was higher in patients with UTIs. In this study, the prevalence of PNE in respondents with UTIs was also significantly higher than that in respondents without UTIs, consistent with the literature [23]. In addition, this study showed that the prevalence of PNE was higher in young adults with frequency/urgency/UI than in those without these symptoms, indicating that bladder dysfunction is a risk factor for PNE in adults. In our study, NMNE had stronger effects on sleep, self-esteem, and depression than MNE. NMNE may often be accompanied by symptoms of frequency and UI, which have more serious impacts on psychology and sleep.

A variety of studies have shown that arousal disorders affect PNE. Hunsballe [24] found that the Y wave was significantly higher during enuresis than during the basal sleep period, indicating that the sleep state increased. Kawawchi et al. [25] reported similar results and suggested that sleep arousal dysfunction is due to the delayed maturation of certain neuronal nuclei in the central nervous system in children. In the present study, the total PSQI, sleep disturbance, sleep medication use, and daytime dysfunction scores were significantly correlated with PNE, indicating that arousal disorder is still a risk factor for PNE in young adults.

It is well known that genetic factors are relevant for PNE. In our previous study in children, 10 years ago, $22.87 \%$ of PNE cases showed a family history of PNE [26]. However, this proportion in the present study increased to $33.89 \%$ (81 of 239 cases) of PNE cases, significantly higher than that found 10 years ago, indicating that heredity is becoming a more prominent risk factor for PNE in young adults. Children with a family history of PNE were further found to be less likely to experience relief with increasing age and to be more likely to continue to experience PNE through adolescence and adulthood. Therefore, early interventions in children with a family history are highly recommended.

Finally, many researchers have reported that PNE often has serious effects on patients and their families, causing serious psychological burdens and life difficulties [27,28]. It is known that PNE is extremely detrimental to a child's psychological and personality formation, as it reduces self-esteem and the daily communication ability. Patients with PNE are prone to anxiety, nervousness, depression, and other negative emotions; this phenomenon becomes increasingly prominent with age [4,7]. It has been reported that the prevalence of anxiety, depression, chronic fatigue, and low self-esteem in adults with NE is significantly higher than that in the general population [7]. The present survey showed similar results. It has been found that patients' self-esteem and depression were significantly related to PNE. The self-esteem scores were lower and the depression scale score was higher in PNE cases than in those without PNE. Since PNE causes psychological disorders, which are also a source of pain, more attention should be paid to PNE treatment and psychological interventions. Doctors must have a sufficient knowledge of psychology to determine whether patients need to be treated by a professional psychiatrist. When university students start their college life, they establish their social environment, and PNE may lead to a decline in their self-esteem and strong embarrassment. Therefore, we should focus more closely on the mental health of university students. In addition, the opposite direction of causality has been suggested - that is, it has been proposed that psychological disorders and stressful experiences could cause PNE [29]. To avoid possible violations of privacy rules, this study did not collect documentation on factors potentially related to psychological outcomes, such as divorce in the family and respondents' financial situation. Therefore, risk factors for mental illness and the relationship between enuresis and psychological disorders should to be further investigated in the future.

It has been reported that one-third of PNE patients have been scolded and punished [30]. Similarly, the present study showed that $34.5 \%$ of patients had been scolded and punished for PNE. It has been verified that punishment is ineffective in the treatment of PNE and may lead to psychological problems [31]. Notably, a result from this study that also warrants attention is that $80 \%$ of PNE patients had not previously sought active treatment. The reasons for the low rate of consultation should be studied further.

Although this study had a large-sample size, it should be kept in mind that the sample was limited to university students. Due to possible violations of privacy rules, information on some factors (e.g., parents' occupations and income) was not collected. The relationship between socioeconomic factors and the prevalence of PNE should be further studied in the future. In our 
study, we only collected information about whether respondents received treatment. However, we did not compare the effects of the treatment, which will be investigated in future research.

The overall prevalence of PNE among Chinese university students was high, and its main risk factors were found to be a family history, daytime voiding symptoms (frequency/urgency/ UI) and lack of treatment during childhood. A family history - indicative of genetic factors - became more closely related to PNE as age increased. PNE showed a significant impact on physical and mental health, as well as sleep. Therefore, on one hand, we should strengthen enuresis awareness and health education and focus more on its psychological impact; on the other hand, the pathogenesis and treatment strategy of enuresis require further exploration in the future.

\section{ACKNOWLEDGEMENTS}

I would like to thank my colleagues, Junqiang Zhao from Xinxiang Medical University and the statistician Prof. Jie Song for their support in conducting this survey.

\section{AUTHOR ORCID}

$\begin{array}{ll}\text { Hui Jie Hu } & 0000-0002-1989-5268 \\ \text { Zhen Wei Zhang } & 0000-0001-8147-1565 \\ \text { Yu Liang } & 0000-0002-2689-9224 \\ \text { Yan Yan Luo } & 0000-0003-0703-5946 \\ \text { Qi Feng Dou } & 0000-0002-6363-2254 \\ \text { Cui Ping Song } & 0000-0002-6095-504 X \\ \text { Hui Min Zhang } & 0000-0003-4775-0397 \\ \text { Ying Zhao } & 0000-0003-3213-7602 \\ \text { Guang Run Tian } & 0000-0001-8716-1031 \\ \text { Ke Zhang } & 0000-0002-3546-9015 \\ \text { Qiu Fang Mao } & 0000-0003-1572-715 X \\ \text { Jing Gui Song } & 0000-0003-3259-4686 \\ \text { Soren Rittig } & 0000-0001-7038-1652 \\ \text { Jian Guo Wen } & 0000-0003-0952-118 X\end{array}$

\section{AUTHOR CONTRIBUTION STATEMENT}

\author{
- Conceptualization: $\mathrm{HJH}$, JGW \\ - Data curation: HJH, ZWZ, YL, HMZ, YZ, GRT, KZ \\ - Formal analysis: $\mathrm{HJH}, \mathrm{ZWZ}$, YL, CPS, QFM \\ - Funding acquisition: JGW
}

- Methodology: HJH, YYL, QF Dou, JGS, JGW

- Project administration: JGW

- Visualization: $\mathrm{HJH}$

-Writing-original draft: $\mathrm{HJH}$

· Writing-review \& editing: SR, JGW

\section{REFERENCES}

1. World Health Organization. Multiaxial classification of child and adolescent psychiatric disorders: The ICD-10 classification of mental and behavioural disorders in children and adolescents. Cambridge (UK): Cambridge University Press; 2008.

2. American Psychiatric Association. Diagnostic and Statistical Manual of Mental Disorders (DSM-5). Washington, DC: American Psychiatric Association; 2013.

3. Austin PF, Bauer SB, Bower W, Chase J, Franco I, Hoebeke P, et al. The standardization of terminology of lower urinary tract function in children and adolescents: update report from the standardization committee of the International Children's Continence Society. Neurourol Urodyn 2016;35:471-81.

4. Yeung CK, Sihoe JD, Sit FK, Bower W, Sreedhar B, Lau J. Characteristics of primary nocturnal enuresis in adults: an epidemiological study. BJU Int 2004;93:341-5.

5. von Gontard A, Cardozo L, Rantell A, Djurhuus JC. Adolescents with nocturnal enuresis and daytime urinary incontinence-How can pediatric and adult care be improved-ICI-RS 2015? Neurourol Urodyn 2017;36:843-9.

6. Van Herzeele C, Walle JV, Dhondt K, Juul KV. Recent advances in managing and understanding enuresis. F1000Res 2017;6:1881.

7. Baek M, Park K, Lee HE, Kang JH, Suh HJ, Kim JH, et al. A nationwide epidemiological study of nocturnal enuresis in Korean adolescents and adults: population based cross sectional study. J Korean Med Sci 2013;28:1065-70.

8. Wen JG, Wang QW, Chen Y, Wen JJ, Liu K. An epidemiological study of primary nocturnal enuresis in Chinese children and adolescents. Eur Urol 2006;49:1107-13.

9. Wang XZ, Wen YB, Shang XP, Wang YH, Li YW, Li TF, et al. The influence of delay elimination communication on the prevalence of primary nocturnal enuresis-a survey from Mainland China. Neurourol Urodyn 2019;38:1423-9.

10. Goessaert AS, Schoenaers B, Opdenakker O, Hoebeke P, Everaert K, Vande Walle J. Long-term follow-up of children with nocturnal enuresis: increased frequency of nocturia in adulthood. J Urol 2014; 191:1866-70.

11. Ninivaggio C, Riese H, Dunivan GC, Jeppson PC, Komesu YM, 
Murata A, et al. One and the same? Nocturnal enuresis and overactive bladder in the female veteran population: evaluation of a large national database. Female Pelvic Med Reconstr Surg 2018;24:30711.

12. Maternik M. Understanding of and misconceptions around monosymptomatic nocturnal enuresis: findings from patient and physician surveys. J Pediatr Urol 2019;15:37.e1-37.e8.

13. Richardson D. Assessment and treatment of nocturnal enuresis in children and young people. Nurs Child Young People 2018;30:407.

14. Tai TT, Tai BT, Chang YJ, Huang KH. Parents have different perceptions of bed-wetting than children from six to 15 years of age. Acta Paediatr 2015;104:e466-72.

15. Jönson Ring I, Nevéus T, Markström A, Arnrup K, Bazargani F. Nocturnal enuresis impaired children's quality of life and friendships. Acta Paediatr 2017;106:806-11.

16. Liu XC, Tang MQ, Hu L. Reliability and validity of the Pittsburgh sleep quality index-Chinese version. Chin J Psychiatry 1996;29:1037.

17. Zung WW. A self-rating depression scale. Arch Gen Psychiatry 1965;12:63-70.

18. Rosenberg M. Society and the adolescent self-image. Princeton (NJ): Princeton University Press; 1965.

19. Wang XD, Wang XL. Rating scales for mental health (updated version). Beijing: Chinese Mental Health Journal Press; 1999. p. 31820.

20. Bogaert G, Stein R, Undre S, Nijman RJM, Quadackers J, ‘t Hoen L, et al. Practical recommendations of the EAU-ESPU guidelines committee for monosymptomatic enuresis-Bedwetting. Neurourol Urodyn 2020;39:489-97.

21. Arena S, Patricolo M. Primary monosymptomatic nocturnal enuresis and associated factors in a referral continence clinic of Abu Dhabi. Pediatr Med Chir 2017;39:150.

22. Hamed A, Yousf F, Hussein MM. Prevalence of nocturnal enuresis and related risk factors in school-age children in Egypt: an epidemiological study. World J Urol 2017;35:459-65.

23. Shah S, Jafri RZ, Mobin K, Mirza R, Nanji K, Jahangir F, et al. Frequency and features of nocturnal enuresis in Pakistani children aged 5 to 16 years based on ICCS criteria: a multi-center cross-sectional study from Karachi, Pakistan. BMC Fam Pract 2018;19:198.

24. Hunsballe JM. Increased delta component in computerized sleep electroencephalographic analysis suggests abnormally deep sleep in primary monosymptomatic nocturnal enuresis. Scand J Urol Nephrol 2000;34:294-302.

25. Kawauchi A, Imada N, Tanaka Y, Minami M, Watanabe H, Shirakawa $S$. Changes in the structure of sleep spindles and delta waves on electroencephalography in patients with nocturnal enuresis. Br J Urol 1998;81 Suppl 3:72-5.

26. Wang QW, Wen JG, Zhang RL, Yang HY, Su J, Liu K, et al. Family and segregation studies: 411 Chinese children with primary nocturnal enuresis. Pediatr Int 2007;49:618-22.

27. Lee D, Dillon BE, Lemack GE. Adult onset nocturnal enuresis: identifying causes, cofactors and impact on quality of life. Low Urin Tract Symptoms 2018;10:292-6.

28. Akhavizadegan H, Locke JA, Stothers L, Kavanagh A. A comprehensive review of adult enuresis. Can Urol Assoc J 2018;13:282-7.

29. Tarcan T, von Gontard A, Apostolidis A, Mosiello G, Abrams P. Can we improve our management of dysfunctional voiding in children and adults: International Consultation on Incontinence Research Society; ICI-RS2018? Neurourol Urodyn 2019;38 Suppl 5: S82-9.

30. Al-Zaben FN, Sehlo MG. Punishment for bedwetting is associated with child depression and reduced quality of life. Child Abuse Negl 2015;43:22-9.

31. Tai TT, Tai BT, Chang YJ, Huang KH. Parental perception and factors associated with treatment strategies for primary nocturnal enuresis. J Pediatr Urol 2017;13:272.e1-272.e8. 